\title{
Retrospective Analysis of Risk Factors and Predictors of Intraoperative Complications in Neuraxial Blocks at Faculdade de Medicina de Botucatu-UNESP
}

\author{
Ivan Dias Fernandes Pereira, TSA ${ }^{1}$, Marcela Miguel Grando ${ }^{2}$, Pedro Thadeu Galvão Vianna, TSA ${ }^{3}$, \\ José Reinaldo Cerqueira Braz, TSA ${ }^{3}$, Yara Marcondes Machado Castiglia, TSA ${ }^{3}$, Luís Antônio Vane, TSA ${ }^{3}$, \\ Norma Sueli Pinheiro Módolo, TSA 4, Paulo do Nascimento Júnior, TSA 4, Rosa Beatriz Amorim 5 , \\ Geraldo Rolim Rodrigues Júnior, TSA ${ }^{5}$, Leandro Gobbo Braz, TSA 5, Eliana Marisa Ganem TSA 4
}

Summary: Pereira IDF, Grando MM, Braz JRC, Castiglia YMM, Vane LA, Módolo NSP, Nascimento Jr P, Amorim RB, Rodrigues Jr GR, Braz LG, Ganem EM - Retrospective Analysis of Risk Factors and Predictors of Intraoperative Complications in Neuraxial Blocks at Faculdade de Medicina de Botucatu-UNESP.

Background and objectives: Cardiovascular changes associated with neuraxial blocks are a cause of concern due to their frequency and because some of them can be considered physiological effects triggered by the sympathetic nervous system blockade. The objective of this study was to evaluate intraoperative cardiovascular complications and predictive factors associated with neuraxial blocks in patients $\geq 18$ years of age undergoing non-obstetric procedures over an 18-year period in a tertiary university hospital - HCFMB-UNESP.

Methods: A retrospective analysis of the following complications was undertaken: hypertension, hypotension, sinus bradycardia, and sinus tachycardia. These complications were correlated with anesthetic technique, physical status (ASA), age, gender, and preoperative co-morbidities. The Tukey test for comparisons among proportions and logistic regression was used for statistical analysis.

Results: 32,554 patients underwent neuraxial blocks. Intraoperative complications mentioned included hypotension ( $n=4,109)$, sinus bradycardia ( $n=1,107)$, sinus tachycardia $(n=601)$, and hypertension $(n=466)$. Hypotension was seen more often in patients undergoing continuous subarachnoid anesthesia $(29.4 \%, O R=2.39), \geq 61$ years of age, and female $(O R=1.27)$.

Conclusions: Intraoperative hypotension and bradycardia were the complications observed more often. Hypotension was related to anesthetic technique (CSA), increased age, and female. Tachycardia and hypertension may not have been directly related to neuraxial blocks.

Keywords: Intraoperative Complications; Anesthesia, Epidural; Anesthesia, Spinal; Arrhythmias, Cardiac; Hypotension; Hypertension.

\section{INTRODUCTION}

Although neuraxial anesthesias are considered technically safe and widely used they are not devoid of risk or side effects ${ }^{1}$.

Hypotension, one of the most common adverse events related to neuraxial blocks caused by the blockade of the sympathetic nervous system ${ }^{2}$ has a variable incidence of $8 \%$ to

Received from Faculdade de Medicina de Botucatu (FMB-UNESP), Brazil.

1. MsC in Anesthesiology from UNESP; Anesthesiologist of the Hospital Universitário da UEM , Hospital do Câncer de Maringá e Hospital Metropolitano de Sarandi

2. Undergraduate medical student at FMB-UNESP; scholarship IC-FAPESP

3. Full Professor at Anesthesiology Department of FMB-UNESP

4. Associate Professor at Anesthesiology Department of FMB-UNESP

5. PhD; Assistant Professor, Physician at Anesthesiology Department of FMB-UNESP

Submitted on January 17, 2010

Approved on February 28, 2011.

Correspondence to:

Dr. Ivan Dias Fernandes Pereira

Rua Jair do Couto Costa, 172 casa 06

Recanto dos Magnatas

87060-625 - Maringá, PR, Brazi

E-mail:idmblh@uol.com.br
$33 \% \%^{1,3-8}$, and such differences can be explained by the different measurement methods and the criteria used to define it.

Sinus bradycardia resulting from cardioinhibitory reflexes activation ${ }^{9,10}$ and cardioaccelerator fibers blockade ${ }^{11}$ occurs when the blockade reaches elevated levels and in young patients with an incidence ranging from $2 \%$ to $13 \%$ in the literature $1,3-8$.

The objective of this retrospective study with non-obstetric adults over an 18-year period in a tertiary university hospital was to identify the incidence and possible causes of hypotension, sinus bradycardia, hypertension, and sinus tachycardia directly or indirectly related to preoperative co-morbidities and neuraxial block.

\section{METHODS}

After approval by the Ethics Committee of the Faculdade de Medicina de Botucatu (UNESP), it was performed a retrospective analysis of neuraxial anesthesia - single-puncture subarachnoid anesthesia (SSA) and continuous subarachnoid anesthesia (CSA), single-puncture epidural anesthesia (SE) and continuous epidural anesthesia (CE), and double block 
(DB) spinal and epidural - in patients 18 years or older undergoing several types of surgical procedures and who recovered in the post anesthetic care unit (PACU) from May 1990 to May 2008.

All patients underwent pre-anesthetic evaluation immediately before urgent and emergency surgeries, and on the day before, during routine pre-anesthetic rounds, in elective procedures. Intraoperative monitoring consisted of a cardioscope, pulse oximetry, and non-invasive blood pressure.

This study used computerized anesthesia records filled out by the resident under supervision of the physician responsible for the anesthesia and catalogued in the database of the Anesthesiology Department (Microsoft Access). Anthropometric data and gender, preoperative co-morbidities, data regarding anesthesia and surgery, and intraoperative complications (list of possible complications on the counter side of the computerized record) were evaluated.

The percentage of patients who presented hypertension (blood pressure above 140/90 $\mathrm{mmHg}$ ), hypotension (a $30 \%$ reduction in the initial blood pressure), sinus bradycardia (heart rate [HR] below $60 \mathrm{bmp}$ ), and sinus tachycardia (HR over $120 \mathrm{bpm}$ ) was evaluated.

These complications were correlated with the anesthetic technique, clinical situation of the patient (physical status according to the American Society of Anesthesiologists), age (18-40 years, 41-60 years, and $\geq 61$ years), gender, and preoperative co-morbidities - hypertension, atrial and ventricular arrhythmias, obesity (BMI > 30), diabetes mellitus, coronary insufficiency $(\mathrm{Cl})$, congestive heart failure (CHF), chronic obs-

Table I - Frequency of Intraoperative Complications

\begin{tabular}{lll}
\hline Total anesthesias & \multicolumn{1}{c}{$\mathbf{N}$} & $\mathbf{( \% )}$ \\
& 32,554 & \\
\hline Hypotension & 4,109 & $(12.6) \mathrm{a}$ \\
Sinus bradycardia & 1,107 & $(3.4) \mathrm{b}$ \\
Sinus tachycardia & 601 & $(1.8) \mathrm{b}$ \\
Hypertension & 466 & $(0.9) \mathrm{b}$ \\
\hline
\end{tabular}

$p<0.0001$ Percentages followed by the same letter did not show statistically significant differences. tructive pulmonary disease (COPD), asthma, renal failure, thyroid diseases, and liver failure. Data regarding preoperative co-morbidities were recorded only from May 1998 onwards.

Tukey test for comparisons among proportions was used in the statistical analysis ${ }^{12}$, adopting a significance level of $p<0.05$ and the logistic regression regarding the variables investigated.

\section{RESULTS}

There were performed 80,660 anesthesias between May 1990 and May 2008 in non-obstetric patients with 18 years of age or older whose records of possible complications were catalogued in the databank of the Anesthesiology Department at the Faculdade de Medicina de Botucatu-UNESP. Of those, 32,554 were of patients who underwent one of the neuraxial techniques investigated. Hypotension was mentioned 4,109 times $(12.6 \%)$, sinus bradycardia $1,107(3.4 \%)$, sinus tachycardia 601 (1.8\%), and hypertension 466 times (0.9\%) (Table I).

Preoperative co-morbidities more prevalent in all age groups included hypertension and diabetes mellitus. Obesity was more common in patients younger than 61 years. Chronic obstructive pulmonary disease, $\mathrm{CHF}, \mathrm{Cl}$, and atrial arrhythmias were also observed in patients with 61 years of age or older (Table II).

Atrial arrhythmias were more frequent in patients aged greater than or equal to 61 years (12.28 times higher than those under the age of 41 years), in patients with ventricular arrhythmias (6.45 times more frequent), and in routine procedures (Table III).

Ventricular arrhythmias were 2.42 times more frequent in patients with physical status ASA II, 11.8 times more frequent in patients ASA III, and 17.34 times more frequent in patients ASA IV when compared to patients ASA I (Table IV).

The majority of patients with hypotension were 61 years of age or older $(p<0.05)$. A statistical significant difference among techniques at different age groups regarding sinus bradycardia and tachycardia, and hypertension were not observed according to Tukey test for comparison of proportions (Table V).

Table II - Frequency of Preoperative Co-morbidities

\begin{tabular}{|c|c|c|c|c|}
\hline \multirow{2}{*}{$\begin{array}{l}\text { Preoperative } \\
\text { Co-morbidities }\end{array}$} & \multirow[b]{2}{*}{18 to 40 years } & \multicolumn{2}{|c|}{ Age N(\%) } & \multirow[t]{2}{*}{$N(\%)$} \\
\hline & & 41 to 60 years & $\geq 61$ years & \\
\hline Hypertension & $1,229(47.3 \%)$ & $1,378(47.8 \%)$ & $2,133(45.5 \%)$ & $4,740(45.6 \%)$ \\
\hline Ventricular arrhythmias & $10(0.4 \%)$ & $26(0.9 \%)$ & $113(2.4 \%)$ & 149 (1.4\%) \\
\hline Obesity & $513(18.2 \%)$ & $333(11.6 \%)$ & $156(3.3 \%)$ & $1,002(9.6 \%)$ \\
\hline Diabetes & 456 (16.2\%) & $550(19.1 \%)$ & $868(18.5 \%)$ & $1,874(18.0 \%)$ \\
\hline Renal failure & $63(2.2 \%)$ & $123(4.3 \%)$ & $191(4.1 \%)$ & 377 (3.6\%) \\
\hline $\mathrm{CHF}$ & $33(1.2 \%)$ & $42(1.5 \%)$ & $271(5.8 \%)$ & $346(3.3 \%)$ \\
\hline Asthma & $291(10.3 \%)$ & $80(2.8 \%)$ & $118(2.5 \%)$ & $489(4.7 \%)$ \\
\hline Thyroid disease & $151(5.4 \%)$ & $105(3.6 \%)$ & $77(1.6 \%)$ & $333(3.2 \%)$ \\
\hline Liver failure & $4(0.1 \%)$ & $10(0.3 \%)$ & $3(0.1 \%)$ & $17(0.2 \%)$ \\
\hline
\end{tabular}

COPD: chronic obstructive respiratory disease, $\mathrm{Cl}$ : coronary insufficiency, $\mathrm{CHF}$ : congestive heart failure. 
Table III - Logistic Regression of Atrial Arrhythmias According the Age Group, Presence of Ventricular Arrhythmias, and Type of Surgery

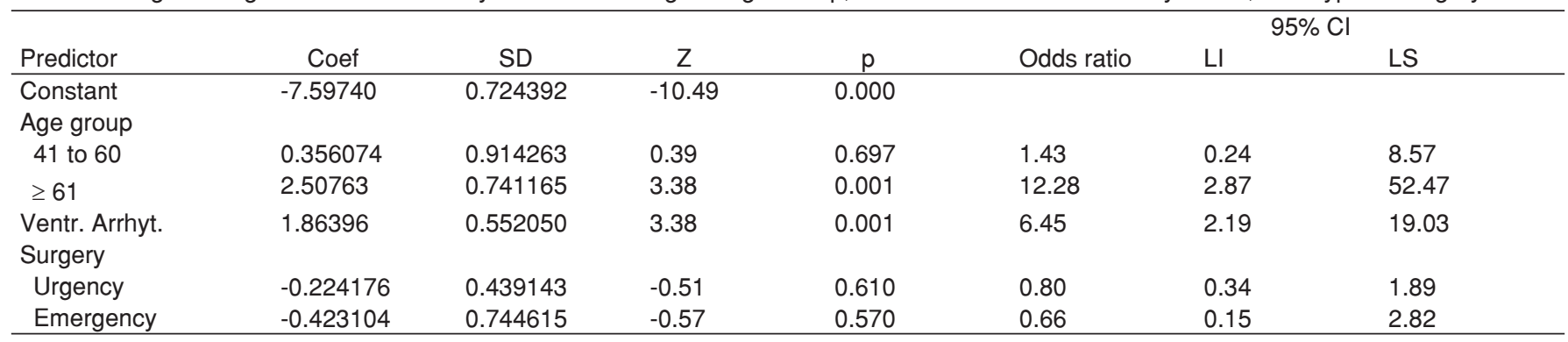

Table IV - Logistic Regression of Ventricular Arrhythmias According to Physical Status

\begin{tabular}{llcccccc}
\hline Predictor & Coef & SD & Z & P & Odds ratio & LI & L5\% Cl \\
\hline Constant & -6.62787 & 0.378214 & -17.52 & 0.000 & & & \\
ASA & & & & & & & \\
II & 0.882036 & 0.458518 & 1.92 & 0.054 & 2.42 & 0.98 & 5.93 \\
III & 2.46775 & 0.442836 & 5.57 & 0.000 & 11.80 & 4.95 & 28.10 \\
IV & 2.85277 & 0.595210 & 4.79 & 0.000 & 17.34 & 5.40 & 55.67 \\
\hline
\end{tabular}

Table V - Total Number of Intraoperative Complications in Different Age Groups

\begin{tabular}{llccc}
\hline & 18 to 40 years & 41 to 60 years & $\geq 61$ years & $p$ \\
Total \# of blockades & 18,910 & 7,153 & 6,491 & $<0.05$ \\
\hline Hypotension (\%) & $2,179(11.5) \mathrm{b}$ & $740(10.3) \mathrm{b}$ & $1,190(18.3) \mathrm{a}$ & $>0.05$ \\
Sinus bradycardia (\%) & $478(2.5)$ & $292(4.1)$ & $(5,2)$ & $>0.05$ \\
Sinus tachycardia (\%) & $392(2.1)$ & $119(1.7)$ & $90(1.4)$ & $>0.05$ \\
Hypertension (\%) & $190(1)$ & $115(1.6)$ & $161(2.5)$ & \\
\hline
\end{tabular}

Percentages followed by the same letter do not show statistically significant differences.

Table VI - Total Number of Intraoperative Complications According to Anesthetic Technique

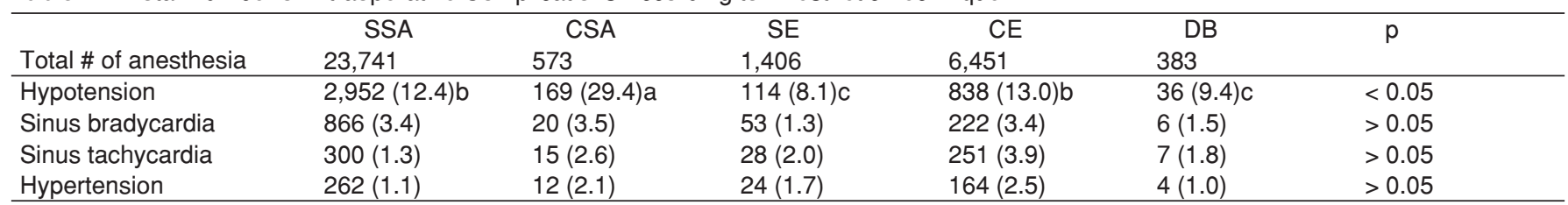

Percentages followed by the same letter do not show statistically significant differences.

Table VII - Logistic Regression of Hypotension According to Age Group, Gender, Physical Status, and Type of Anesthesia

\begin{tabular}{|c|c|c|c|c|c|c|c|}
\hline \multirow[b]{2}{*}{ Predictor } & \multirow[b]{2}{*}{ Coef } & \multirow[b]{2}{*}{ SD } & \multirow[b]{2}{*}{ Z } & \multirow[b]{2}{*}{$p$} & \multirow[b]{2}{*}{ Odds ratio } & \multicolumn{2}{|c|}{$95 \% \mathrm{Cl}$} \\
\hline & & & & & & $\mathrm{LI}$ & LS \\
\hline$\geq 61$ & 1.02822 & 0.0931459 & 11.04 & 0.000 & 2.80 & 2.33 & 3.36 \\
\hline $\begin{array}{l}\text { Gender } \\
\text { ASA }\end{array}$ & 0.238046 & 0.0589611 & 4.04 & 0.000 & 1.27 & 1.13 & 1.42 \\
\hline III & 0.784356 & 0.160355 & 4.89 & 0.000 & 2.19 & 1.60 & 3.00 \\
\hline Anesthesia & & & & & & & \\
\hline C. spinal & 0.872870 & 0.122768 & 7.11 & 0.000 & 2.39 & 1.88 & 3.04 \\
\hline S. epi. & 0.334834 & 0.191395 & 1.75 & 0.080 & 1.40 & 0.96 & 2.03 \\
\hline C. epi & 0.493791 & 0.0756657 & 6.53 & 0.000 & 1.64 & 1.41 & 1.90 \\
\hline Double-block & 0.0916701 & 0.250009 & 0.37 & 0.714 & 1.10 & 0.67 & 1.79 \\
\hline
\end{tabular}


RETROSPECTIVE ANALYSIS OF RISK FACTORS AND PREDICTORS OF INTRAOPERATIVE COMPLICATIONS IN NEURAXIAL BLOCKS AT FACULDADE DE MEDICINA DE BOTUCATU-UNESP

Table VIII - Logistic Regression of Hypertension According to Age Group, Obesity, and Type of Anesthesia

\begin{tabular}{|c|c|c|c|c|c|c|c|}
\hline \multirow[b]{2}{*}{ Predictor } & \multirow[b]{2}{*}{ Coef } & \multirow[b]{2}{*}{ SA } & \multirow[b]{2}{*}{ Z } & \multirow[b]{2}{*}{$\mathrm{p}$} & \multicolumn{3}{|c|}{$95 \% \mathrm{Cl}$} \\
\hline & & & & & Odds ratio & $\mathrm{LI}$ & LS \\
\hline \multicolumn{8}{|l|}{ Age group } \\
\hline$\geq 61$ & 2.46533 & 0.398433 & 6.19 & 0.000 & 11.77 & 5.39 & 25.69 \\
\hline Obesity & 1.00445 & 0.258873 & 3.88 & 0.000 & 2.73 & 1.64 & 4.54 \\
\hline \multicolumn{8}{|l|}{ Anesthesia } \\
\hline C. epi. & 0.463575 & 0.209080 & 2.22 & 0.027 & 1.59 & 1.06 & 2.39 \\
\hline Double-block & 0.165909 & 0.721525 & 0.23 & 0.818 & 1.18 & 0.29 & 4.86 \\
\hline
\end{tabular}

Table IX - Logistic Regression of Sinus Bradycardia According to Age Group, Gender, Physical Status, Obesity, and Type of Surgery

\begin{tabular}{|c|c|c|c|c|c|c|c|}
\hline \multirow[b]{2}{*}{ Predictor } & \multirow[b]{2}{*}{ Coef } & \multirow[b]{2}{*}{ SD } & \multirow[b]{2}{*}{ Z } & \multirow[b]{2}{*}{$\mathrm{p}$} & \multicolumn{3}{|c|}{$95 \% \mathrm{Cl}$} \\
\hline & & & & & Odds ratio & $\mathrm{LI}$ & LS \\
\hline \multicolumn{8}{|l|}{ Age group } \\
\hline$\geq 61$ & 0.606928 & 0.168119 & 3.61 & 0.000 & 1.83 & 1.32 & 2.55 \\
\hline Gender & -0.295043 & 0.116857 & -2.52 & 0.012 & 0.74 & 0.59 & 0.94 \\
\hline \multicolumn{8}{|l|}{ ASA } \\
\hline IV & -0.632408 & 0.470933 & -1.34 & 0.179 & 0.53 & 0.21 & 1.34 \\
\hline Obesity & 0.413543 & 0.236173 & 1.75 & 0.080 & 1.51 & 0.95 & 2.40 \\
\hline \multicolumn{8}{|l|}{ Surgery } \\
\hline Urgency & -0.729069 & 0.148700 & -4.90 & 0.000 & 0.48 & 0.36 & 0.65 \\
\hline Emergency & -1.60543 & 0.416576 & -3.85 & 0.000 & 0.20 & 0.09 & 0.45 \\
\hline
\end{tabular}

Table X - Logistic Regression of Sinus Tachycardia According to Age Group, Physical Status, Type of Surgery, and Type Anesthesia

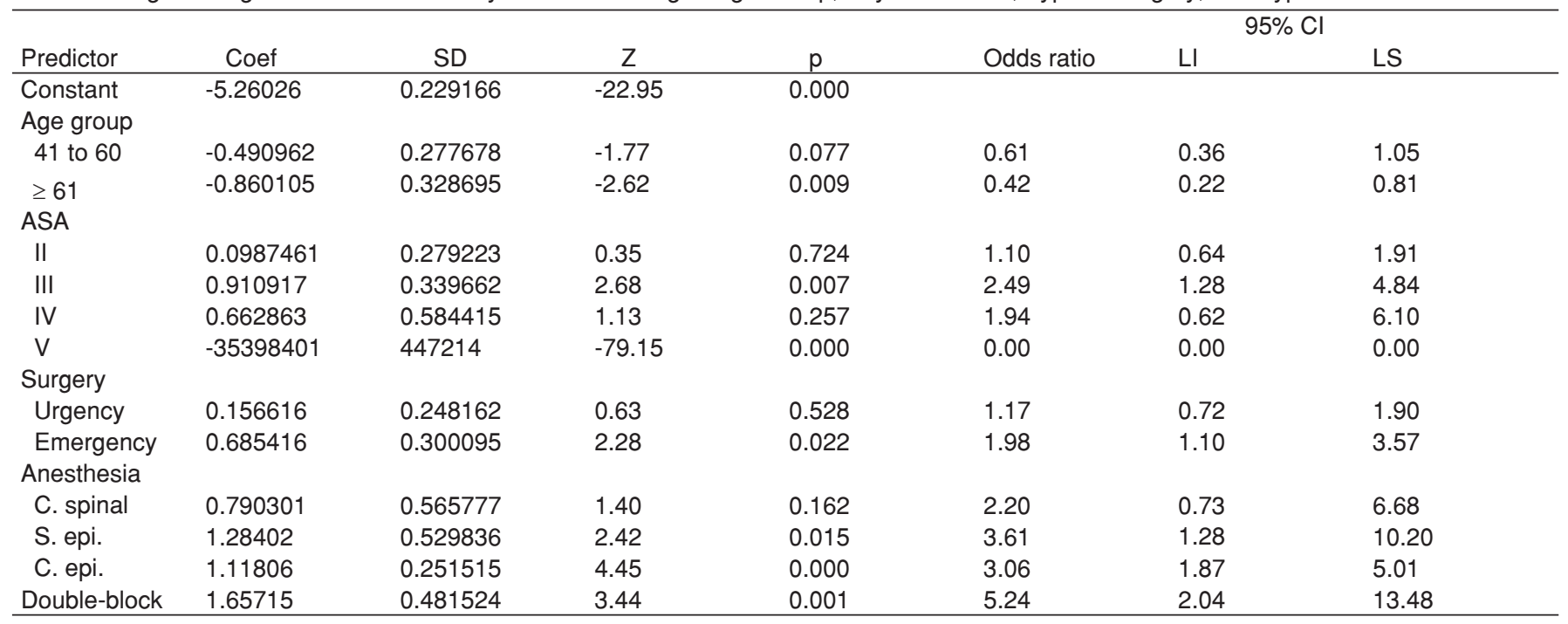


Hypotension was more frequent in patients undergoing CSA (29.4\%), followed by SSA (12.4\%), which was similar to CE $(13 \%)(p<0.05)$. The incidence of sinus bradycardia and tachycardia, as well as hypertension, was similar in the different techniques of neuraxial blockade (Tukey test) (Table VI).

Logistic regression showed that variables that associated with hypotension were age group, gender, and type of anesthesia. The chance of an individual developing hypotension was 1.51 times higher at ages between 41 and 60 years and 2.80 times higher in the age group $\geq 61$ years when compared to patients younger than 41 years of age. The chance of a female developing hypotension was 1.27 times higher than males. Regarding the anesthesia, the chance was 2.39 times higher with continuous subarachnoid anesthesia, 1.40 times higher with simple epidural, 1.64 times higher with continuous epidural, and 1.10 times higher with double block when compared to simple subarachnoid anesthesia (Table VII).

Regarding hypertension, the results of logistic regression showed an association with age group, obesity, and type of anesthesia. The chance of a patient developing hypertension was 7.59 times greater if he/she was 41 to 60 years of age, and 11.77 times greater if he/she was $\geq 61$ years of age when compared to patients younger than 41 years of age. Obese patients had a 2.73 times greater chance of developing hypertension than non-obese patients. Regarding the type of anesthesia, the chance was 1.18 times greater with continuous subarachnoid anesthesia, 2.30 times greater with simple epidural anesthesia, 1.59 times greater with continuous epidural anesthesia, and 1.18 times greater with double block when compared with simple subarachnoid (Table VIII).

Variables that showed an association with sinus bradycardia included age group, gender, physical status (ASA), obesity, and type of surgery (routine, urgent, emergency). The chance of an individual developing sinus bradycardia was 1.40 times greater if he/se was 41 to 60 years of age, and 1.83 if he/she was $\geq 61$ years of age when compared to individuals younger than 41 years of age. Males were 1.35 times more likely to develop sinus bradycardia than females.

Regarding physical status the chance of an individual developing sinus bradycardia was 0.81 times lower if he/she was classified as ASA II, 0.56 times lower as ASA III, and 0.53 times lower as ASA IV when compared to those who were ASA I. The chance was 1.51 times greater if the individual was obese. Regarding the type of surgery the chance was 0.48 times lower in case of urgency, and 0.20 times lower in case of emergency when compared to routine surgeries (Table IX).

Variables that were associated with sinus tachycardia included age group, physical status ASA, type of surgery, and type of anesthesia. The chance of an individual developing sinus tachycardia was 0.61 times lower if he/she was 41 to 61 years of age, and 0.42 times lower in the age group $\geq 61$ years when compared to those younger than 41 years. The chance of individuals developing sinus tachycardia was 1.10 times greater if they were classified as ASA II, 2.49 times greater in ASA III, and 1.94 times greater in ASA IV when compared to those classified as ASA I. Regarding the type of surgery the chance was 1.17 times greater in urgent surgeries and 1.98 times greater in emergency surgeries when compared to routine surgeries. Regarding the type of anesthesia the chance was 2.20 times greater with continuous subarachnoid anesthesia, 3.61 times greater with simple epidural, 3.06 times greater with continuous epidural, and 5.24 times greater with double block when compared with simple subarachnoid anesthesia (Table X).

\section{DISCUSSION}

The results of this study showed that hypotension was the most common adverse event in neuraxial anesthesia with an incidence of $12.6 \%$.

In the literature ${ }^{1,3-8}$, the incidence of hypotension ranges from $8 \%$ to $33 \%$. This variation is due to the different classification methods and criteria used to define hypotension. The definitions used more often include systolic blood pressure (SBP) below a specific level (in general, 80 or $90 \mathrm{mmHg}$ ), or a predetermined reduction in initial SBP or mean arterial pressure (MAP) (usually $30 \%$ ), or even a fast decline in blood pressure (such as $30 \%$ of baseline BP or $30 \mathrm{mmHg}$ in five minutes), without taking into consideration an absolute value.

It has been described that the parameters that best correlate with hypotension include the level of the blockade $\geq T 5$, age $\geq 40$ years, SBP of $120 \mathrm{mmHg}$, association of subarachnoid anesthesia with general anesthesia, puncture above L2-L3, addition of phenylephrine to the local anesthetic (LA), chronic alcohol consumption, prior hypertension, elevated body mass index, and urgent surgeries ${ }^{1,6}$.

Tarkkila et al. ${ }^{13}$ observed and incidence of $15.3 \%$, which was similar to the results found in the present study, and they defined hypotension as a $30 \%$ reduction of baseline levels or SBP lower than $85 \mathrm{mmHg}$.

Autonomic blockade triggered by neuraxial block promotes dilation of resistance and capacitance vessels ${ }^{13-16}$, resulting in reduction of venous return, right cardiac chambers filling pressures, systemic vascular resistance, and cardiac output 17-19. Redistribution of the central blood volume to the splanchnic circulation and the lower extremities reduces blood pressure ${ }^{20}$.

Elevated plasma concentrations of LA ${ }^{21,22}$, which cause a dose-dependent myocardial depression, adrenaline through its stimulating action in $\beta 2$ receptors, and clonidine ${ }^{23}$, due to its agonist action in $\alpha 2$ adrenergic receptors could also be involved in the genesis of hypotension.

In the present study the chance of hypotension was 2.32 times greater with CSA, 1.64 times greater with CE, 1.40 times greater with SP, and 1.10 times greater with $\mathrm{DB}$ when compared with SSA. These results differ from those of other authors ${ }^{24-26}$, who have reported a lower incidence of hypotension and use of vasopressors after CSA when compared to SSA. The continuous technique, which enable titration of $\mathrm{AL}$ doses and better control of anesthetic blockade extension reduces the risk of hypotension and is a good indication in elderly patients $24,27,28$.

Intraoperative hypotension was more common in patients 61 years of age or older undergoing CSA, CE, and SSA 
(18.3\%). The most frequent co-morbidities affecting this age group included hypertension, diabetes mellitus, atrial arrhythmias, coronary insufficiency, and heart failure.

The reduction in cardiac reserve and changes in baroreceptor and sympathetic nervous system responses make hypotension and bradycardia the most common complications in elderly patients undergoing neuraxial blocks ${ }^{29,30}$.

Patients with hypertension were twice more likely to develop hypotension than non-hypertensive patients ${ }^{31}$. In the elderly and in those with elevated body mass index, the risk of hypotension is increased ${ }^{13}$.

Continuous subarachnoid anesthesia was more often in patients older than 61 years of age. Although the literature describes that hemodynamic changes are les marked with this technique ${ }^{26,28}$, in this study a greater occurrence of hypertension and diabetes mellitus was observed in this age group. A higher incidence of atrial arrhythmias, coronary insufficiency and congestive heart failure was also observed in this age group, which might have been an additional factor for the development of cardiocirculatory instability that is usually associated with autonomic blockade contributing to the higher incidence of hypotension.

In this study, sinus bradycardia was the second more common complication affecting 1,107 patients, corresponding to an incidence of $3.4 \%$. Several studies have reported incidences ranging from $2 \%$ to $13 \%{ }^{1,3-8}$.

Bradycardia results from the blockade of the sympathetic nervous system with the consequent predominance of parasympathetic tonus decreased venous return ${ }^{32}$. The blockade of cardioaccelerator fibers, which originate from $\mathrm{T} 1$ to $\mathrm{T} 4$, is part of the triggering mechanism of bradycardia ${ }^{11}$.

Several reflex responses secondary to decreased venous return are involved in the genesis of bradycardia ${ }^{9}$, such as the reflex that involves stretching of sinus pacemaker cells, baroreceptors on the right atrial wall and in the atrium-vena cava junction, and mechanoreceptors on the inferior portion of ventricular walls (Belzold-Jarisch reflex) ${ }^{10}$. Excessive sedation, pre-existing autonomic dysfunction, cardiac block, vasovagal reaction ${ }^{33}$, and athletic heart syndrome ${ }^{34}$ may also give rise to bradycardia during neuraxial anesthesia.

Several studies ${ }^{35-37}$ have demonstrated that a baseline heart rate below $60 \mathrm{bpm}$, physical status ASA I, treatment with beta-blockers, sensorial level above T6, age lower than 50 years, and prolonged PR interval are risk factors for the development of bradycardia.

Patients who underwent subarachnoid anesthesia (single puncture or continuous anesthesia) and continuous epidural anesthesia had the higher incidence of bradycardia, as well as patients 61 years of age or older.

The chance of developing sinus bradycardia in the present study was greater in patients ASA I when compared to ASA II, III, and IV, most likely due to the fact that vagal tonus is more pronounced in younger patients ${ }^{1}$. On the other hand, the chance was greater in patients with 61 years of age or older $(\mathrm{OR}=1.83$ ) when compared to patients younger than 41 years due to the higher incidence of co-morbidities in the elderly.
In this study, obese patients had a greater chance of developing bradycardia $(\mathrm{OR}=1.51)$. Although the extent of sensory block correlates with the volume of CSF in the lumbar region and not with the $\mathrm{BMI}$, elderly patients could have more elevated levels of blockade since the increase in abdominal pressure would compress the subarachnoid space, therefore reducing the volume of CSF in this region. However, the risk of relevant hypotension in obese patients does not seem to be related with the more elevated level of the blockade ${ }^{38}$.

An association between male gender and sinus bradycardia was observed $(O R=1.35)$, which was also reported by other authors who observed that in males the activation of vagal response after central volume reduction triggered by the epidural block was more intense 39 .

In this study, bradycardia was more frequent in routine anesthesia than in urgent or emergency ones. These data differ from those reported in the literature, in which sinus bradycardia is more frequent in patients undergoing urgent and emergency anesthesia that might have pre-existent diseases non-diagnosed or inadequately treated. In emergency procedures, intraoperative instability may result from increased sympathetic activity and blood losses associated with trauma 6 .

Although moderate sinus bradycardia is well tolerated the sudden and intense reduction in heart rate during subarachnoid and epidural anesthesia may progress to asystole ${ }^{37,40}$.

Therefore, there is a concern in diagnosing and treating adequately sinus bradycardia, considered an indicative sign of impending vascular collapse, as described in a study with 40,640 subarachnoid anesthesias in which patients developed bradycardia immediately before cardiac arrest ${ }^{41}$.

Sinus tachycardia and hypertension are not complications directly related to neuraxial anesthesia, and since this was a retrospective study it is difficult to correlate them exclusively to an anesthetic technique. The results may be a consequence of preoperative hypertension, stress, prolonged surgery with partial blockade regression and pain, sympathomimetic and parasympathomimetic drugs used to treat hypotension and bradycardia, insufflation or deflation of the tourniquet (in surgeries in which there was the need of garroting the lower limbs) ${ }^{42}$, and increased sympathetic reflex above the level of the blockade when subarachnoid anesthesia was restricted to the lower thoracic or lumbar dermatomes ${ }^{43}$.

This study has several limitations in which, especially due to its retrospective nature, it was not always possible to know whether the complication was due to the anesthetic technique, surgery, associated diseases, or even exacerbated effects of the drugs used to correct other complications in the intraoperative period.

However, since this study involved an elevated number of anesthesias, it was important to trace the profile of cardiovascular complication related to preoperative co-morbidities and neuraxial blocks performed in a tertiary university hospital (Hospital das Clínicas da FMB-UNSEP) over an 18-year period. 